\title{
Dynamic Analysis of Tendon Driven Robotic Mechanisms
}

\author{
Jyh-Jone Lee* and Yueh-Hua Lee \\ Department of Mechanical Engineering \\ National Taiwan University \\ Taipei, Taiwan, 10617 \\ Republic of China \\ e-mail: jjlee@ccms.ntu.edu.tw \\ Received 8 March 2002; accepted 11 December 2002
}

\begin{abstract}
In this paper, a systematic methodology for the dynamic analysis of tendon-driven robotic mechanisms is presented. The approach utilizes the recursive Newton-Euler equations to compute the kinematic and dynamic equations of all links that locate on the transmission line of a tendon-driven robotic mechanism. The inertias of the intermediate links in the mechanism are taken into account. It is shown that the dynamic equations can be established in a recursive manner from the end-effector links toward the proximal links and can be solved at the proximal end without the need of solving the simultaneous system equations. The joint reaction forces and the tension in each segment of tendon can be also obtained. The methodology can be applied to both endless and open-ended tendon drives. (c) 2003 Wiley Periodicals, Inc.
\end{abstract}

\section{INTRODUCTION}

For decades, the study of tendon-driven systems has remained interesting to researchers in many fields such as design of dexterous mechanical hands, parallel cable-suspended manipulators, and teleoperating robots. The main advantages of using tendon drives lie in that actuators can be controlled from the outset of system, resulting in a compact and lightweight design of the mechanical device. In addition, a well designed tendon transmission system has little

*To whom all correspondence should be addressed. backlash. However, the use of a tendon transmission may bring some drawbacks, such as extra mechanical components, the creation of frictions and hysteresis loss upon the system, resulting in the complexity of the mechanical system.

For this reason, there has been a wide interest in the study of mechanics of tendon transmission systems. The mechanics of a multi-pulley train with flexible drives was studied by Chen. ${ }^{1}$ The kinematics and statics of articulated tendon-driven robotic mechanisms were addressed by Morecki et al., ${ }^{2}$ Sulisbury, ${ }^{3}$ and Tsai and Lee. ${ }^{4}$ However, the dynamics of such a mechanical system was accomplished on an 
individual basis ${ }^{5,6}$ or performed on single DOF devices. ${ }^{7,8}$ Hollars and Cannon ${ }^{5}$ took into account tendon flexibility and pulley inertia and analyzed the dynamics of two-degree-of-freedom (DOF) manipulators. Prisco and Bergamasco ${ }^{6}$ modeled the dynamics of the $2 \mathrm{~N}$ type of tendon-driven manipulator using the Lagrangian equation. Instead of using the Lagrangian approach, we present a systematic methodology for the dynamic analysis of tendon-driven manipulators. The methodology incorporates the recursive Newton and Euler formulation such that the joint forces, tendon forces and equations of motion can be both explicitly and systematically obtained. This paper is organized as follows. First, we review the kinematics of tendon-driven robotic mechanisms. Then, we present the dynamic analysis of the links. Finally, we show how the forward and inverse dynamics can be analyzed systematically using the proposed approach. Several examples will also be used to demonstrate the procedure.

\section{ASSUMPTION}

In a previous paper, ${ }^{4}$ some general assumptions about the structural characteristics have been introduced for the kinematic and static analysis of tendondriven manipulators. In what follows, we shall introduce two additional assumptions for tendon-driven manipulators regarding the dynamic analysis.

(i) Tendons are lightweight such that the weight/ inertia, flexural bending, and shear effects of tendons will not be included.

(ii) For sakes of clarity and simplicity, the frictions in the pulley bearings and other moving parts of the transmission are not included. Nevertheless, these terms may be added in the modeling where the force control is important.

Tendon drives can generally be classified into two types of routing ${ }^{4,9}$ as (1) the open-ended type and (2) the endless type of routing. In the open-ended tendon routing, as shown in Figure 1(a), one end of the tendon is fixed to a moving link to be controlled while the other end is attached to a driving actuator. From the moving link to the driving actuator, each tendon routing forms a transmission line. A unique characteristic of such open-ended tendon drives is that tendons transmit the forces in a unidirectional sense. On the other hand, the routing of the endless tendons is shown in Figure 1(b) in which two pulleys of constant

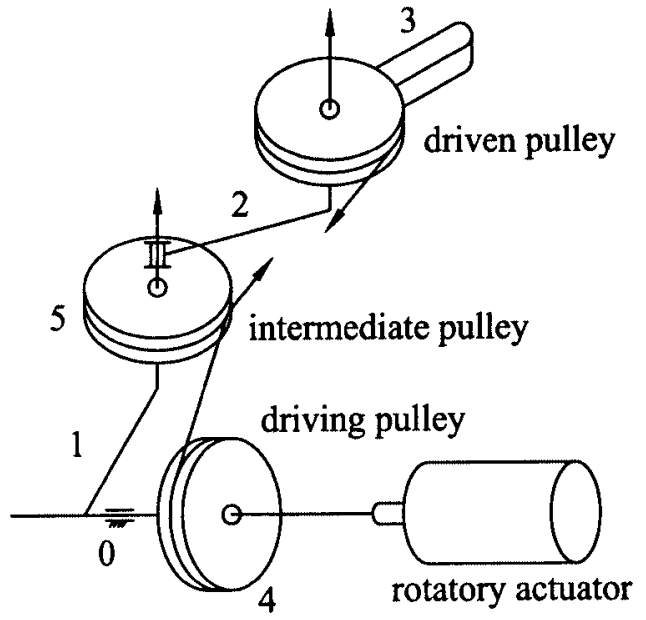

(a)

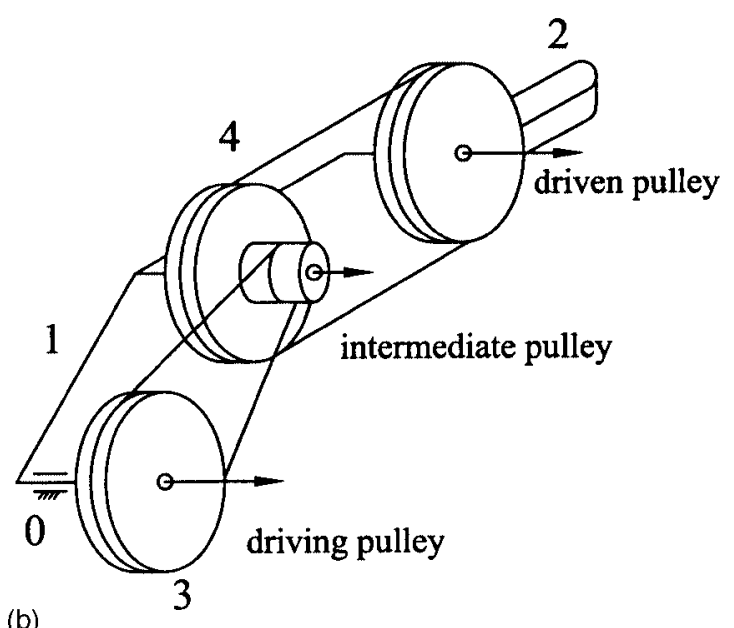

Figure 1. (a) Open-ended type tendon routing. (b) Endless type tendon routing.

center distance are wrapped around by an endless tendon. The driven pulley is attached to a link to be controlled and the driving pulley is installed on a rotary actuator or fixed to a driven pulley of prior pulley train stage. From the driven link to the driving actuator, the tendon-and-pulley also forms a transmission line. In an endless tendon drive, the pulley can be driven in both directions. One side of the tendon will be under higher tension while the other side is under lower tension.

It can be noted that after the removal of tendons and intermediate/idle pulleys, the tendon-driven robotic mechanism becomes a serial type open-loop chain. We call the links that constitute the open-loop chain the primary links, and all other links the intermediate links. An intermediate link is said to be carried by a primary link $i$ if it is connected to link $i$ by 
a revolute joint. As shown in Figure 1(a), links 0, 1, 2 and 3 are the primary links, and links 4 and 5 are the intermediate links. Intermediate links 4 and 5 are carried by primary link 1 .

\section{KINEMATICS OF PRIMARY LINKS}

To facilitate the analysis, we sequentially number each primary link from the base to the distal link as 0 to $n$. Meanwhile, a local coordinate system $\left(x_{i}, y_{i}, z_{i}\right)$ is attached to the distal joint of link $i$ according to the Denavit and Hartenberg (D-H) convention. ${ }^{10}$ Let $\theta_{i, i-1}$ be the joint angle from $x_{i-1}$ axis to $x_{i}$ axis, $a_{i, i-1}$ be the offset distance between $z_{i-1}$ and $z_{i}$ axes, and $\alpha_{i, i-1}$ be the twist angle. Then, the coordinate transformation from the $i$ th coordinate $\mathbf{X}_{i}$ to the $(i-1)$ th coordinate $\mathbf{X}_{i-1}$ can be written as

$$
\mathbf{X}_{i-1}={ }^{i-1} \mathbf{A}_{i} \mathbf{X}_{i}
$$

where matrix ${ }^{i-1} \mathbf{A}_{i}$ is given as

$$
\left[\begin{array}{cccc}
C \theta_{i, i-1} & -C \alpha_{i, i-1} S \theta_{i, i-1} & S \alpha_{i, i-1} S \theta_{i, i-1} & a_{i, i-1} C \theta_{i, i-1} \\
S \theta_{i, i-1} & C \alpha_{i, i-1} C \theta_{i, i-1} & -S \alpha_{i, i-1} C \theta_{i, i-1} & a_{i, i-1} S \theta_{i, i-1} \\
0 & S \alpha_{i, i-1} & C \alpha_{i, i-1} & d_{i, i-1} \\
0 & 0 & 0 & 1
\end{array}\right]
$$

and where $C \theta_{i, i-1}=\operatorname{Cos}\left(\theta_{i, i-1}\right), S \theta_{i, i-1}=\operatorname{Sin}\left(\theta_{i, i-1}\right)$, $C \alpha_{i, i-1}=\operatorname{Cos}\left(\alpha_{i, i-1}\right), S \alpha_{i, i-1}=\operatorname{Sin}\left(\alpha_{i, i-1}\right)$.

Since the primary links form an open-loop chain, their velocities and accelerations can be derived from the forward recursive method, ${ }^{11}$ computing from the proximal moving link toward the end-effector link, as

$$
\begin{gathered}
{ }^{i} \boldsymbol{\omega}_{i}={ }^{i} \mathbf{R}_{i-1}\left({ }^{i-1} \boldsymbol{\omega}_{i-1}+\dot{\theta}_{i, i-1}{ }^{i-1} \mathbf{Z}_{i-1}\right) \\
{ }^{i} \dot{\boldsymbol{\omega}}_{i}={ }^{i} \mathbf{R}_{i-1}\left({ }^{i-1} \dot{\boldsymbol{\omega}}_{i-1}+\ddot{\theta}_{i, i-1}{ }^{i-1} \mathbf{Z}_{i-1}+{ }^{i-1} \boldsymbol{\omega}_{i-1}\right. \\
\left.\times \dot{\theta}_{i, i-1}{ }^{i-1} \mathbf{Z}_{i-1}\right), \\
{ }^{i} \mathbf{V}_{i}={ }^{i} \mathbf{R}_{i-1}{ }^{i-1} \mathbf{V}_{i-1}+{ }^{i} \boldsymbol{\omega}_{i} \times{ }^{i} \mathbf{P}_{i, i-1} \\
{ }^{i} \dot{\mathbf{V}}_{i}={ }^{i} \dot{\boldsymbol{\omega}}_{i} \times{ }^{i} \mathbf{P}_{i, i-1}+{ }^{i} \mathbf{R}_{i-1}{ }^{i-1} \dot{\mathbf{V}}_{i-1}+{ }^{i} \boldsymbol{\omega}_{i} \times\left({ }^{i} \boldsymbol{\omega}_{i} \times{ }^{i} \mathbf{P}_{i, i-1}\right), \\
{ }^{i} \dot{\mathbf{V}}_{i c}={ }^{i} \dot{\boldsymbol{\omega}}_{i} \times{ }^{i} \mathbf{r}_{i c}+{ }^{i} \dot{\mathbf{V}}_{i}+{ }^{i} \boldsymbol{\omega}_{i} \times\left({ }^{i} \boldsymbol{\omega}_{i} \times{ }^{i} \mathbf{r}_{i c}\right)
\end{gathered}
$$

where $\omega_{i}$ is the angular velocity vector of link $i, \mathbf{V}_{i}$ is the velocity vector of the origin $O_{i}, \mathbf{V}_{i c}$ is the velocity of the center of mass of link $i, \mathbf{r}_{i c}$ is position vector defined from the mass center of link $i$ to $O_{i}$, ${ }^{i-1} \mathbf{Z}_{i-1}=[0,0,1]^{T}$ is a unit vector defined along the $\mathbf{Z}_{i-1}$ axis, ${ }^{i} \mathbf{R}_{i-1}$ is the transpose of the upper left 3 $\times 3$ submatrix of ${ }^{i-1} \mathbf{A}_{i}$, and ${ }^{i} \mathbf{P}_{i, i-1}$ is the vector as

$$
{ }^{i} \mathbf{P}_{i, i-1}=\left[a_{i, i-1}, d_{i, i-1} S \alpha_{i, i-1}, d_{i, i-1} C \alpha_{i, i-1}\right]^{T} .
$$

\section{KINEMATICS OF INTERMEDIATE LINKS}

A local coordinate system $\left(x_{j}, y_{j}, z_{j}\right)$ is defined for each intermediate link according to the D-H convention. Consider an intermediate link $j$ which is located on the primary link $i$. The coordinate transformation matrix from the $j$ th coordinate system to the $i$ th coordinate is given by

$$
{ }^{i} \mathbf{B}_{j}=\left[\begin{array}{cccc}
C \theta_{j, i} & -C \alpha_{j, i} S \theta_{j, i} & S \alpha_{j, i} S \theta_{j, i} & a_{j, i} C \theta_{j, i} \\
S \theta_{j, i} & C \alpha_{j, i} C \theta_{j, i} & -S \alpha_{j, i} C \theta_{j, i} & a_{j, i} S \theta_{j, i} \\
0 & S \alpha_{j, i} & C \alpha_{j, i} & d_{j, i} \\
0 & 0 & 0 & 1
\end{array}\right] .
$$

Suppose $j, k$, and $i$ constitute a simple tendonand-pulley train where links $j, k$ are pulleys and link $i$ is the carrier. Then, the relative rotations of links $j$ and $k$ with respect to link $i$ can be written as

$$
\theta_{j, i}=\rho_{k j} \theta_{k, i}
$$

where $\rho_{k j}$ is the pulley ratio for the pulley pairs mounted on link $i$, positive according to a positive rotation of pulley $k$ resulting in a positive rotation of pulley $j$ about their predefined axes of rotation.

Suppose links $i, j$, and $k$ are coaxial. Then, the relative motion among these links can be related by the coaxial condition as 


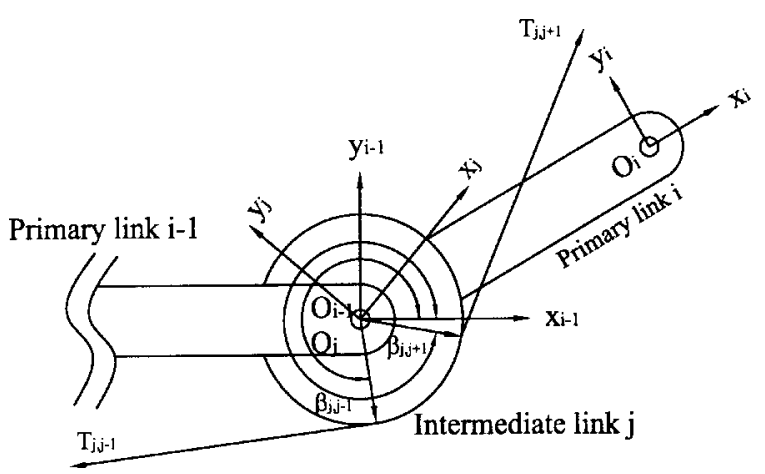

Figure 2. A typical intermediate link $j$ carried by a primary link $i-1$.

$$
\theta_{i, k}=\theta_{i, j}+\theta_{j, k}
$$

The velocity and acceleration of intermediate link j can be also obtained by modifying Eqs. (2)-(6) as

$$
\begin{gathered}
{ }^{j} \boldsymbol{\omega}_{j}={ }^{j} \mathbf{Q}_{i}{ }^{i} \boldsymbol{\omega}_{i}+\dot{\theta}_{j, i}{ }^{j} \mathbf{Z}_{j} \\
{ }^{j} \dot{\boldsymbol{\omega}}_{j}={ }^{j} \mathbf{Q}_{i}{ }^{i} \dot{\boldsymbol{\omega}}_{i}+\ddot{\theta}_{j, i}{ }^{j} \mathbf{Z}_{j}+{ }^{j} \mathbf{Q}_{i}{ }^{i} \boldsymbol{\omega}_{i} \times \dot{\boldsymbol{\theta}}_{j, i}{ }^{j} \mathbf{Z}_{j} \\
{ }^{j} \mathbf{V}_{j}={ }^{j} \mathbf{Q}_{i}{ }^{i} \mathbf{V}_{i}+{ }^{j} \boldsymbol{\omega}_{j} \times{ }^{j} \mathbf{P}_{j, i}, \\
{ }^{j} \dot{\mathbf{V}}_{j}={ }^{j} \dot{\boldsymbol{\omega}}_{j} \times{ }^{j} \mathbf{P}_{j, i}+{ }^{j} \mathbf{Q}_{i}{ }^{i} \dot{\mathbf{V}}_{i}+{ }^{j} \mathbf{Q}_{i}{ }^{i} \boldsymbol{\omega}_{i} \times(12) \\
{ }^{j} \mathbf{Q}_{i}{ }^{i} \boldsymbol{\omega}_{j} \times{ }^{j}{ }^{j} \mathbf{P}_{j, i} \times\left({ }^{j}{ }^{j} \mathbf{P}_{j, i}+{ }^{j}{ }_{\mathbf{r}_{j c}}\right)+{ }^{j} \mathbf{Q}_{i}{ }^{i} \dot{\mathbf{V}}_{i}+{ }^{j} \mathbf{Q}_{i}{ }^{i} \boldsymbol{\omega}_{i} \times\left[{ }^{j} \mathbf{Q}_{i}{ }^{i} \boldsymbol{\omega}_{i}\right. \\
\left.\times\left({ }^{j} \mathbf{P}_{j, i}+{ }^{j} \mathbf{r}_{j c}\right)\right],
\end{gathered}
$$

where ${ }^{j} \mathbf{Q}_{i}$ is the transpose of the upper left $3 \times 3$ submatrix of ${ }^{i} \mathbf{B}_{j}, \mathbf{r}_{j c}$ is the position vector defined from mass center of links $j$ to $O_{j}$, and ${ }^{j} \mathbf{P}_{j, i}$ can be written as

$$
{ }^{j} \mathbf{P}_{j, i}=\left[a_{j, i}, d_{j, i} S \alpha_{j, i}, d_{j, i} C \alpha_{j, i}\right]^{T} .
$$

\section{TENDON FORCES}

Since each transmission line begins from the primary link and ends at the link driven by the rotary actuator, the tendon may route through one or more intermediate links. Consider the intermediate link $j$ on the distal joint of a primary link $i-1$ as shown in Figure 2. Let the carrier of the pulley pair $(j, j+1)$ be the pri- mary link $i$, and the carrier of the pulley pair $(j, j$ -1 ) be the primary link $i-1$. Also, let the portion of the tendon connecting intermediate links $j$ and $j+1$ be $T_{j, j+1}$, and the other portion connecting links $j$ and $j-1$ be $T_{j, j-1}$.

For a general tendon-pulley pair, the tendon will engage with the pulley at a constant direction with respect to the carrier. In addition, for the pulley to run in both directions, the direction of tendon acting on the pulley should be tangential to the pitch circle of the pulley plane. As shown in Figure 2, define the acting angle of the tendon $\mathbf{T}_{j, j+1}$ as $\beta_{j, j+1}$ measured from the $x$-axis of coordinate system on link $i-1$ in a righthand rule direction, and similarly the acting angle of tendon $\mathbf{T}_{j, j-1}$ as $\beta_{j, j-1}$ measured from the same system. Then, we can write the tendon force in the $(i-1)$ th coordinate system as

$$
{ }^{i-1} \mathbf{T}_{j, j+1}=T_{j, j+1}\left[-\operatorname{Sin} \beta_{j, j+1},+\operatorname{Cos} \beta_{j, j+1}, 0\right]^{T},
$$

where $T_{j, j+1}$ is the magnitude of $\mathbf{T}_{j, j+1}$. Similarly the tendon force ${ }^{i-1} \mathbf{T}_{j, j-1}$ can also be obtained.

The tendon forces can be transformed to the $j$ th coordinate system on link $j$ by multiplying a rotational matrix ${ }^{j} \mathbf{R}_{i-1}$ :

$$
{ }^{j} \mathbf{T}_{j, j-1}={ }^{j} \mathbf{R}_{i-1}{ }^{i-1} \mathbf{T}_{j, j-1} \cdot
$$

From Eq. (18), it can be seen that the direction of the tendon will depend on the acting angle and the $z$ component of $\mathbf{T}_{j, j-1}$ on the apex location. Only one unknown magnitude is necessarily specified for the tendon force $\mathbf{T}_{j, j-1}$. In the absence of frictional and flexural effects of tendon routing, the tendon forces across the intermediate link $j$ will be differentiated due to the inertia effect of the intermediate link.

\section{DYNAMICS ANALYSIS}

For a given mechanism in a known state of motion, the force and moments exerted by different links on each other can be determined via Newton and Euler's equations. While solving the dynamics using Newton and Euler's equations, three force- and three moment-balance equations can be established for each link of the mechanism. Usually, the total number of balance equations of the system will be equal to that of the unknown variables. To solve the simultaneous equations in an efficient way, rather than solving all the unknowns simultaneously, one tries to 


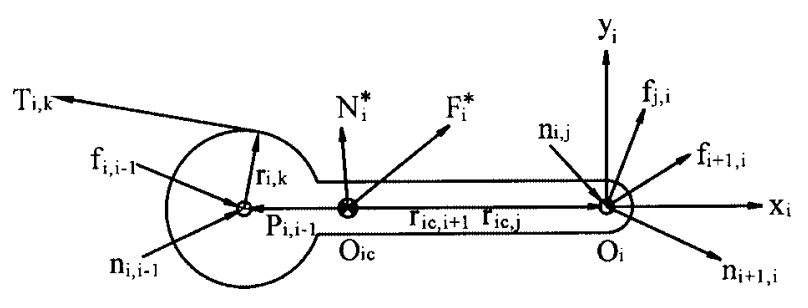

Figure 3. A typical primary link.

solve them in a recursive manner such that an individual link with six unknowns or a small subgroup of links with fewer unknowns are identified and solved. Once these equations are solved, the balance equations for the consecutive link or subgroup of links may become solvable. This procedure continues until all the unknowns in the system are solved. However, in tendon-driven drives, the solution condition will not be the same as above. The unknowns in any step will always outnumber the balance equations due to the redundancy of tendon forces. Hence, the procedure of solution may be similar in recursiveness except that the joint forces will first be expressed in tendon forces, then the balance equations need to be solved simultaneously for $n$ primary links in terms of tendon forces that are connected to the primary links. After the tendon forces are solved, the joint forces can then be determined by back substituting the tendon forces into the balance equations.

\section{DYNAMICS OF PRIMARY LINKS}

Figure 3 shows the free body diagram of a primary link $i$ which connects to link $(i-1)$ at joint $(i-1)$ and link $(i+1)$ at joint $i$. Assume that primary link $i$ is driven by the $k$ th tendon or more. The force and moment balance equations for link $i$ can be represented in the following recursive forms:

$$
{ }^{i} \mathbf{f}_{i, i-1}+\sum_{k}{ }^{i} \mathbf{T}_{i, k}={ }^{i} \mathbf{F}_{i}^{*}+{ }^{i} \mathbf{f}_{i+1, i}+\sum_{j}{ }^{i} \mathbf{f}_{j, i}
$$

and

$$
\begin{aligned}
{ }^{i} \mathbf{n}_{i, i-1}+{ }^{i} \mathbf{P}_{i c} \times{ }^{i} \mathbf{f}_{i, i-1}+\sum_{k}\left[\left({ }^{i} \mathbf{r}_{i, k}+{ }^{i} \mathbf{P}_{i c}\right) \times{ }^{i} \mathbf{T}_{i, k}\right] \\
={ }^{i} \mathbf{N}_{i}^{*}+{ }^{i} \mathbf{n}_{i+1, i}+{ }^{i} \mathbf{r}_{i c} \times{ }^{i} \mathbf{f}_{i+1, i}-\sum_{j}{ }^{i} \mathbf{n}_{i, j} \\
+\sum_{j}\left({ }^{i} \mathbf{r}_{i c} \times{ }^{i} \mathbf{f}_{j, i}\right),
\end{aligned}
$$

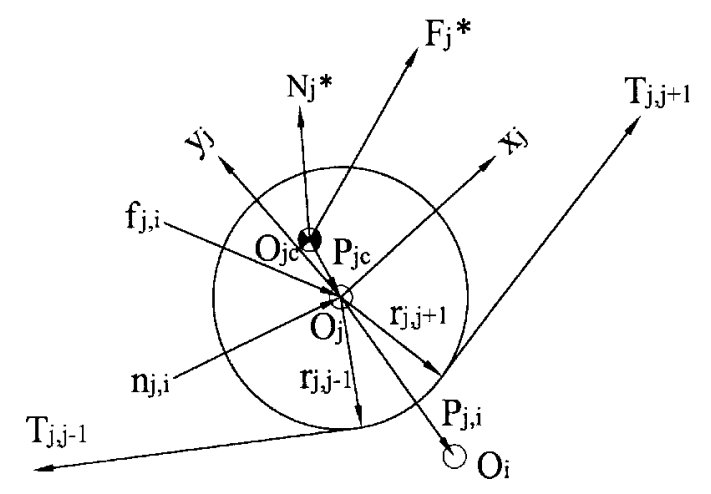

Figure 4. A typical intermediate link $j$.

where ${ }^{i} \mathbf{F}_{i}^{*}$ and ${ }^{i} \mathbf{N}_{i}^{*}$ are the inertia force and moment vectors of primary link $i$ and can be obtained as

$$
\begin{gathered}
{ }^{i} \mathbf{F}_{i}^{*}=m_{i}{ }^{i} \dot{\mathbf{V}}_{i c}, \\
{ }^{i} \mathbf{N}_{i}^{*}={ }^{c} \mathbf{I}_{i}{ }^{i} \dot{\boldsymbol{\omega}}_{i}+{ }^{i} \boldsymbol{\omega}_{i} \times\left({ }^{c} \mathbf{I}_{i}{ }^{i} \boldsymbol{\omega}_{i}\right),
\end{gathered}
$$

where $m_{i}$ is the mass of link $i$ and ${ }^{c} \mathbf{I}_{i}$ is the inertia tensor of link $i$ with respect to a center of mass coordinate system which has the same orientation as the $i$ th coordinate system.

The vector terms on the right-hand side of Eqs. (19) and (20), $\mathbf{f}_{i+1, i}$ and $\mathbf{n}_{i+1, i}$, are computed from the balance equations of the preceding link while $\mathbf{f}_{j, i}$ and $\mathbf{n}_{j, i}$ are the forces and moments from intermediate links. For the end-effector link, these vectors represent the end-effector output force and moment. The left-hand side of Eqs. (19) and (20) contain all the unknown force and moment vectors: $\mathbf{f}_{i, i-1}, \mathbf{n}_{i, i-1}$ and $\mathbf{T}_{i, k}$. It can be seen that $\mathbf{f}_{i, i-1}, \mathbf{n}_{i, i-1}$ and $\mathbf{T}_{i, k}$ always constitute more than six scalar unknowns. Hence they cannot be solved one link at a time.

\section{DYNAMICS OF THE INTERMEDIATE LINKS}

Referring to Figure 4, the force and moment balance equations for an intermediate link $j$ can be written as

$$
{ }^{j} \mathbf{f}_{j, i}={ }^{i} \mathbf{F}_{j}^{*}-{ }^{j} \mathbf{T}_{j, j-1}-{ }^{j} \mathbf{T}_{j, j+1}
$$

and

$$
\begin{aligned}
{ }^{j} \mathbf{n}_{j, i}+\left({ }^{j} \mathbf{r}_{j, j-1}+{ }^{j} \mathbf{r}_{j c}\right) \times{ }^{j} \mathbf{T}_{j, j-1} \\
\quad{ }^{={ }^{j}} \mathbf{N}_{j}^{*}-{ }^{j} \mathbf{r}_{j c} \times{ }^{j} \mathbf{f}_{j, i}-\left({ }^{j} \mathbf{r}_{j, j+1}+{ }^{j} \mathbf{r}_{j c}\right) \times{ }^{j} \mathbf{T}_{j, j+1} .
\end{aligned}
$$




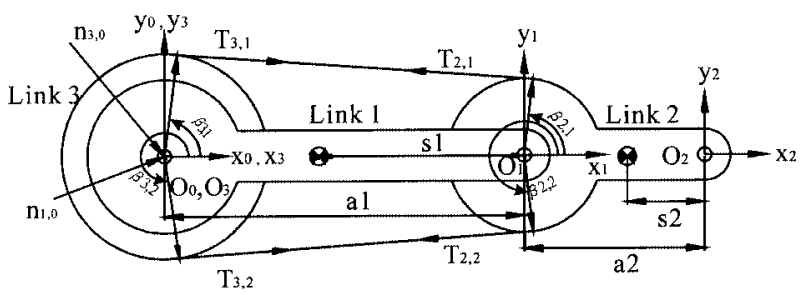

Figure 5. A 2-DOF robotic mechanism driven by endless tendons.

Equation (24a) can be simplified as

$$
{ }^{j} \mathbf{n}_{j, i}+{ }^{j} \mathbf{r}_{j, j-1} \times{ }^{j} \mathbf{T}_{j, j-1}={ }^{j} \mathbf{N}_{j}^{*}-{ }^{j} \mathbf{r}_{j, j+1} \times{ }^{j} \mathbf{T}_{j, j+1}-{ }^{j} \mathbf{r}_{j c} \times{ }^{j} \mathbf{F}_{j}^{*},
$$

where ${ }^{j} \mathbf{F}_{j}^{*}$ and ${ }^{j} \mathbf{N}_{j}^{*}$ are the inertia force and moment of link $j$, and can be computed similarly by Eqs. (21) and (22).

The tendon force $T_{j, j+1}$ in Eqs. (23) and (24b) can be computed from the moment balance equation of the upper level intermediate link. Once the joint forces and the moment of upper level links are solved, the unknown forces and moments in Eqs. (23) and $(24 \mathrm{~b})$ can be solved associated with the unknown forces and moments in the primary link. We also note that any vector defined in its local coordinate system on the intermediate link can be related to the coordinate system of its corresponding primary link by Eqs. (17) and (18).

We have derived the basic equations which are required for the dynamic analysis of articulated, tendon-driven robotic devices. In what follows, we describe a systematic procedure for the derivation of dynamic equations of the articulated, tendon-driven robotic devices. Three examples will be used to illustrate the procedure.

\section{Example 1}

Figure 5 shows a 2-DOF robotic mechanism driven by endless tendons. Two motors are to be installed at the joint axis 0 and drive links 1 and 3, respectively.

From the figure, we have $\left.{ }^{1} \mathbf{P}_{1,0}=\left[a_{1}, 0,0\right]\right]^{T},{ }^{2} \mathbf{P}_{2,1}$ $=\left[a_{2}, 0,0\right]^{T}$ and ${ }^{3} \mathbf{P}_{3,0}=[0,0,0]^{T}$. Let ${ }^{1} \mathbf{r}_{1 c}=\left[s_{1}, 0,0\right]^{T}$, ${ }^{2} \mathbf{r}_{2 c}=\left[s_{2}, 0,0\right]^{T}$ and ${ }^{3} \mathbf{r}_{3 c}=[0,0,0]^{T}$. Then, the necessary kinematics of primary links 1,2 and intermediate link 3 can be computed from Eqs. (2)-(6) and (11)-(15). The position vectors required for Eq. (19), (20), (23) and (24) are ${ }^{1} \mathbf{P}_{1 c}=\left[-\left(a_{1}-s_{1}\right), 0,0\right]^{T},{ }^{2} \mathbf{P}_{2 c}=\left[-\left(a_{2}\right.\right.$ $\left.\left.-s_{2}\right), 0,0\right]^{T}$. In addition, the directions of tendon forces and their corresponding positions are identified as

$$
\begin{gathered}
{ }^{2} \mathbf{T}_{2,1}={ }^{2} \mathbf{R}_{1} T_{2,1}\left[-S \beta_{2,1}, C \beta_{2,1}, 0\right]^{T}, \\
{ }^{2} \mathbf{T}_{2,2}={ }^{2} \mathbf{R}_{1} T_{2,2}\left[S \beta_{2,2},-C \beta_{2,2}, 0\right]^{T}, \\
{ }^{3} \mathbf{T}_{3,1}={ }^{3} \mathbf{R}_{1} T_{3,1}\left[S \beta_{3,1},-C \beta_{3,1}, 0\right]^{T}, \\
{ }^{3} \mathbf{T}_{3,2}={ }^{3} \mathbf{R}_{1} T_{3,2}\left[-S \beta_{3,2}, C \beta_{3,2}, 0\right]^{T}, \\
{ }^{2} \mathbf{r}_{21}={ }^{2} \mathbf{R}_{1} r_{2}\left[C \beta_{2,1}, S \beta_{2,1}, 0\right]^{T}, \\
{ }^{2} \mathbf{r}_{22}={ }^{2} \mathbf{R}_{1} r_{2}\left[C \beta_{2,2}, S \beta_{2,2}, 0\right]^{T}, \\
{ }^{3} \mathbf{r}_{31}={ }^{3} \mathbf{R}_{1} r_{3}\left[C \beta_{3,1}, S \beta_{3,1}, 0\right]^{T}
\end{gathered}
$$

and

$$
{ }^{3} \mathbf{r}_{32}={ }^{3} \mathbf{R}_{1} r_{3}\left[C \beta_{3,2}, S \beta_{3,2}, 0\right]^{T} .
$$

We note that $T_{2,1}=T_{3,1}, T_{2,2}=T_{3,2}$ in the absence of frictions along tendons.

From Eq. (9), angular displacement of intermediate link can be expressed in terms of the joint displacement as

$$
\theta_{3,1}=\rho_{23} \theta_{2,1} .
$$

From the coaxial condition, ${ }^{4}$ the angular displacement of base pulleys can be related to the joint angles as

$$
\left[\begin{array}{c}
\theta_{3,0} \\
\theta_{1,0}
\end{array}\right]=\left[\begin{array}{cc}
1 & \rho_{23} \\
1 & 0
\end{array}\right]\left[\begin{array}{c}
\theta_{1,0} \\
\theta_{2,1}
\end{array}\right]
$$

The dynamic loading on the bearing of link 2 and tendon forces can be determined respectively from the force and moment balance equations about the mass center of link 2 as

$$
\begin{aligned}
{ }^{2} \mathbf{f}_{2,1}= & { }^{2} \mathbf{F}_{2}^{*}-{ }^{2} \mathbf{T}_{2,1}-{ }^{2} \mathbf{T}_{2,2}, \\
r_{2}\left(T_{2,1}-T_{2,2}\right)= & { }_{2} I_{2}\left(\ddot{\theta}_{1,0}+\ddot{\theta}_{2,1}\right)+m_{2}\left(a_{2}-s_{2}\right) \\
& \times\left(a_{1}\left(S \theta_{2,1} \dot{\theta}_{1,0}^{2}+C \theta_{2,1} \ddot{\theta}_{1,0}\right)\right. \\
& \left.+\left(a_{2}-s_{2}\right)\left(\ddot{\theta}_{1,0}+\ddot{\theta}_{2,1}\right)\right),
\end{aligned}
$$




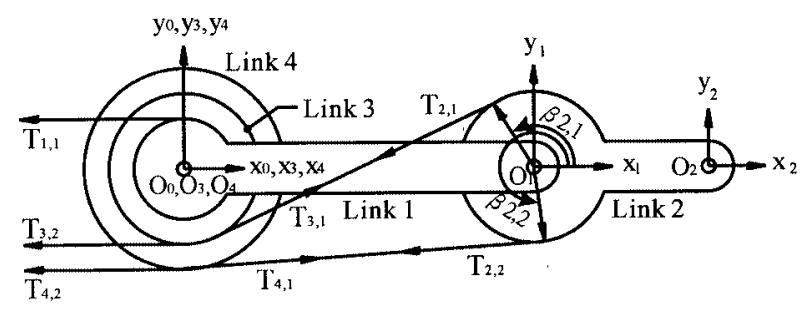

Figure 6. A 2-DOF robotic mechanism driven by openended tendons.

where ${ }^{2} \mathbf{F}_{2}^{*}$ is the inertia force acting on link 2. Similarly, the actual forces $\left(\mathbf{f}_{3,0}^{*}, \mathbf{n}_{3,0}^{*}\right)$ and $\left(\mathbf{f}_{1,0}^{*}, \mathbf{n}_{1,0}^{*}\right)$ can be determined form the balance equations about links 3 and 1 , respectively. The motor torques required for links 3 and 1 are

$$
\begin{aligned}
& \mathbf{n}_{3,0}^{*}=r_{3}\left(T_{2,1}-T_{2,2}\right)+{ }^{c} I_{3}\left(\ddot{\theta}_{1,0}+\rho_{23} \ddot{\theta}_{2,1}\right) \\
\mathbf{n}_{1,0}^{*}= & \left(r_{1}-r_{2}\right)\left(T_{2,2}-T_{2,1}\right)-a_{1}\left(( a _ { 2 } - s _ { 2 } ) S \theta _ { 2 , 1 } m _ { 2 } \left(\dot{\theta}_{1,0}^{2}\right.\right. \\
& \left.+\dot{\theta}_{2,1}^{2}\right)-\left(a_{2}-s_{2}\right) C \theta_{2,1} m_{2}\left(\ddot{\theta}_{1,0}-\ddot{\theta}_{2,1}\right)+2 m_{3} s_{1} \ddot{\theta}_{1,0} \\
& +\left({ }^{c} I_{1}+a_{1}^{2}\left(m_{2}+m_{3}\right)+m_{3} s_{1}^{2}\right) \ddot{\theta}_{1,0} .
\end{aligned}
$$

By substituting Eq. (28) into Eqs. (29) and (30), tendon forces $\left(T_{2,1}-T_{2,2}\right)$ can be eliminated in the moment balance equations (29) and (30). Hence, the motor torques will be affected by the dynamic characteristics of links regardless of the tendon tension. However, the bearing forces $\mathbf{f}_{2,1}, \mathbf{f}_{3,0}$, and $\mathbf{f}_{1,0}$ will be affected by the tension in tendons as seen in Eq. (27). Usually, the pretensioning of tendons in an endless routing cannot be actively adjusted. Thus, once the pretensioning of tendons is adjusted, the static loading on bearing is also determined.

\section{Example 2}

Let the mechanism in Figure 5 be driven by three open-ended tendons, as shown in Figure 6 . Then it is necessary to determine the tendon forces among the pulleys. The kinematics of links is computed as in Example 1 . The direction of tendon forces and their positions are

$$
\begin{aligned}
& { }^{2} \mathbf{T}_{2,1}={ }^{2} \mathbf{R}_{1} T_{2,1}\left[-S \beta_{2,1}, C \beta_{2,1}, 0\right]^{T}, \\
& { }^{2} \mathbf{T}_{2,2}={ }^{2} \mathbf{R}_{1} T_{2,2}\left[S \beta_{2,2},-C \beta_{2,2}, 0\right]^{T},
\end{aligned}
$$

$$
\begin{aligned}
& { }^{3} \mathbf{T}_{3,1}={ }^{3} \mathbf{R}_{1} T_{3,1}\left[-S \beta_{3,1}, C \beta_{3,1}, 0\right]^{T}, \\
& { }^{3} \mathbf{T}_{3,2}={ }^{3} \mathbf{R}_{0} T_{3,2}\left[S \beta_{3,2},-C \beta_{3,2}, 0\right]^{T}, \\
& { }^{4} \mathbf{T}_{4,1}={ }^{4} \mathbf{R}_{1} T_{4,1}\left[-S \beta_{4,1}, C \beta_{4,1}, 0\right]^{T}, \\
& { }^{4} \mathbf{T}_{4,2}={ }^{4} \mathbf{R}_{0} T_{4,2}\left[S \beta_{4,2},-C \beta_{4,2}, 0\right]^{T}, \\
& { }^{1} \mathbf{T}_{1,1}={ }^{1} \mathbf{R}_{0} T_{1,1}\left[-S \beta_{1,1}, C \beta_{1,1}, 0\right]^{T},
\end{aligned}
$$

and

$$
\begin{gathered}
{ }^{2} \mathbf{r}_{2,1}={ }^{2} \mathbf{R}_{1} r_{2}\left[C \beta_{2,1}, S \beta_{2,1}, 0\right]^{T}, \\
{ }^{2} \mathbf{r}_{2,2}={ }^{2} \mathbf{R}_{1} r_{2}\left[C \beta_{2,2}, S \beta_{2,2}, 0\right]^{T}, \\
{ }^{3} \mathbf{r}_{3,1}={ }^{3} \mathbf{R}_{1} r_{3}\left[C \beta_{3,1}, S \beta_{3,1}, 0\right]^{T}
\end{gathered}
$$

and

$$
\begin{gathered}
{ }^{3} \mathbf{r}_{3,2}={ }^{3} \mathbf{R}_{0} r_{3}\left[C \beta_{3,2}, S \beta_{3,2}, 0\right]^{T}, \\
{ }^{4} \mathbf{r}_{4,1}={ }^{4} \mathbf{R}_{1} r_{4}\left[C \beta_{4,1}, S \beta_{4,1}, 0\right]^{T}, \\
{ }^{4} \mathbf{r}_{4,2}={ }^{4} \mathbf{R}_{0} r_{4}\left[C \beta_{4,2}, S \beta_{4,2}, 0\right]^{T}
\end{gathered}
$$

and

$$
{ }^{1} \mathbf{r}_{1,1}={ }^{1} \mathbf{R}_{0} r_{1}\left[C \beta_{1,1}, S \beta_{1,1}, 0\right]^{T} .
$$

Note that $T_{3,1}=T_{2,1}, T_{4,1}=T_{2,2}$.

The displacement relation between the joint angles and the base pulley angles is

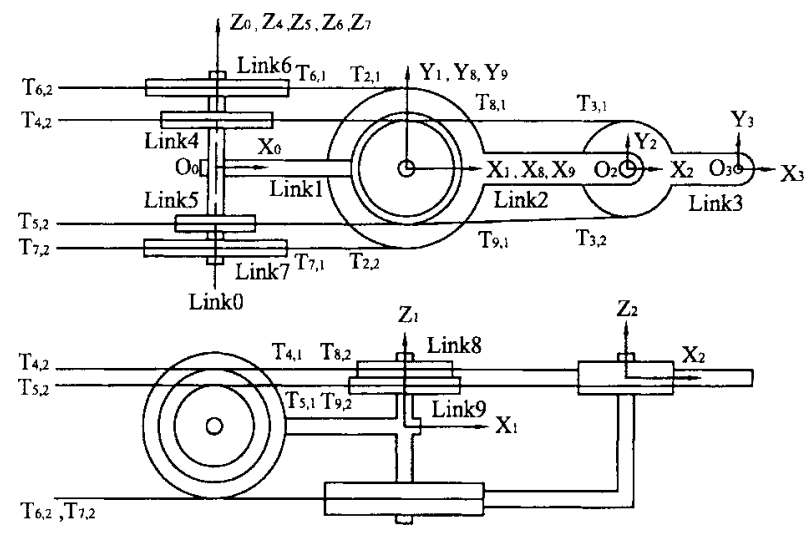

Figure 7. The Stanford-JPL finger. 
Table I. Tendon forces vectors used in Example 3.

$\begin{array}{ll}{ }^{3} \mathbf{T}_{3,1}={ }^{3} \mathbf{R}_{2} T_{3,1}\left[-S \beta_{3,1}, C \beta_{3,1}, 0\right]^{T} & \\ { }^{8} \mathbf{T}_{8,1}={ }^{8} \mathbf{R}_{2} T_{8,1}\left[\beta_{8,1}-C \beta_{8,1}, 0\right]^{T} & { }^{3} \mathbf{T}_{3,2}={ }^{3} \mathbf{R}_{2} T_{3,2}\left[S \beta_{3,2},-C \beta_{3,2}, 0\right]^{T} \\ { }_{9}^{9} \mathbf{T}_{9,1}={ }^{9} \mathbf{R}_{2} T_{9,1}\left[-S \beta_{9,1}, C \beta_{9,1}, 0\right]^{T} & { }^{8} \mathbf{T}_{8,2}={ }^{8} \mathbf{R}_{1} T_{8,2}[-1,0,0]^{T} \\ { }^{2} \mathbf{T}_{2,1}={ }^{2} \mathbf{R}_{1} T_{2,1}[-1,0,0]^{T} & { }^{9} \mathbf{T}_{9,2}={ }^{9} \mathbf{R}_{1} T_{9,2}[-1,0,0]_{T}^{T} \\ { }^{4} \mathbf{T}_{4,1}={ }^{4} \mathbf{R}_{1} T_{4,1}[1,0,0]^{T} & { }^{2} \mathbf{T}_{2,2}={ }^{2} \mathbf{R}_{1} T_{2,2}[-1,0,0]^{T} \\ { }^{5} \mathbf{T}_{5,1}={ }^{5} \mathbf{R}_{1} T_{5,1}[1,0,0]^{T} & { }^{4} \mathbf{T}_{4,2}={ }^{4} \mathbf{R}_{0} T_{4,2}\left[-S \beta_{4,2}, C \beta_{4,2}, 0\right]^{T} \\ { }^{6} \mathbf{T}_{6,1}={ }^{6} \mathbf{R}_{1} T_{6,1}[1,0,0]^{T} & { }^{5} \mathbf{T}_{5,2}={ }^{5} \mathbf{R}_{0} T_{5,2}\left[-S \beta_{5,2}, C \beta_{5,2}, 0\right]^{T} \\ { }^{7} \mathbf{T}_{7,1}={ }^{7} \mathbf{R}_{1} T_{7,1}[1,0,0]^{T} & { }^{6} \mathbf{T}_{6,2}={ }^{6} \mathbf{R}_{0} T_{6,2}\left[S \beta_{6,2},-C \beta_{6,2}, 0\right]^{T} \\ \end{array}$

$$
\left[\begin{array}{l}
\theta_{1,0} \\
\theta_{3,0} \\
\theta_{4,0}
\end{array}\right]=\left[\begin{array}{cc}
1 & 0 \\
1 & \rho_{23} \\
1 & \rho_{24}
\end{array}\right]\left[\begin{array}{l}
\theta_{1,0} \\
\theta_{2,1}
\end{array}\right] .
$$

Since there are four links in the devices, we can get eight balance equations as follows:

$$
\begin{gathered}
\mathbf{f}_{2,1}+\mathbf{T}_{2,1}+\mathbf{T}_{2,2}=\mathbf{F}_{2}^{*} \\
\mathbf{P}_{2 c} \times \mathbf{f}_{2,1}+\left(\mathbf{r}_{2,1}+\mathbf{P}_{2 c}\right) \times \mathbf{T}_{2,1}+\left(\mathbf{r}_{2,2}+\mathbf{P}_{2 c}\right) \times \mathbf{T}_{2,2}=\mathbf{N}_{2}^{*} \\
\mathbf{f}_{3,0}+\mathbf{T}_{3,1}+\mathbf{T}_{3,2}=\mathbf{F}_{3}^{*} \\
\mathbf{P}_{3 c} \times \mathbf{f}_{3,0}+\left(\mathbf{r}_{3,1}+\mathbf{P}_{3 c}\right) \times \mathbf{T}_{3,1}+\left(\mathbf{r}_{3,2}+\mathbf{P}_{3 c}\right) \times \mathbf{T}_{3,2}=\mathbf{N}_{3}^{*}, \\
\mathbf{f}_{4,0}+\mathbf{T}_{4,1}+\mathbf{T}_{4,2}=\mathbf{F}_{4}^{*}, \\
\mathbf{P}_{4 c} \times \mathbf{f}_{4,0}+\left(\mathbf{r}_{4,1}+\mathbf{P}_{4 c}\right) \times \mathbf{T}_{4,1}+\left(\mathbf{r}_{4,2}+\mathbf{P}_{4 c}\right) \times \mathbf{T}_{4,2}=\mathbf{N}_{4}^{*}, \\
\mathbf{f}_{1,0}+\mathbf{T}_{1,1}=\mathbf{F}_{1}^{*}+\mathbf{f}_{2,1} \\
\mathbf{P}_{1 c} \times \mathbf{f}_{1,0}+\left(\mathbf{r}_{1,1}+\mathbf{P}_{1 c}\right) \times \mathbf{T}_{1,1}=\mathbf{N}_{1}^{*}-\mathbf{r}_{1 c} \times \mathbf{f}_{2,1}
\end{gathered}
$$

Substituting the force $\mathbf{f}_{2,1}$ in (32a) into (32b) and representing the tendon forces $\mathbf{T}_{3,1}$ and $\mathbf{T}_{4,1}$ in Eqs. (32d) and (32f) in terms of $T_{3,2}$ and $T_{4,2}$ and again substituting into $(32 \mathrm{~b})$ yields

$$
r_{2} T_{3,2}-r_{2} T_{4,2}=b_{1}
$$

where $\quad b_{1}={ }^{c} I_{2}\left(\ddot{\theta}_{1,0}+\ddot{\theta}_{2,1}\right)+m_{2}\left(a_{2}-s_{2}\right)\left(a_{1}\left(S \theta_{2,1} \dot{\theta}_{1,0}^{2}\right.\right.$ $\left.\left.+C \theta_{2,1} \ddot{\theta}_{1,0}\right)-\left(a_{2}-s_{2}\right)\left(\ddot{\theta}_{1,0}+\ddot{\theta}_{2,1}\right)\right)\left(r_{2} / r_{3}\right)^{c} I_{3}\left(\ddot{\theta}_{1,0}+\rho_{23} \ddot{\theta}_{2,1}\right)$ $+\left(r_{2} / r_{4}\right){ }^{c} I_{4}\left(\ddot{\theta}_{1,0}+\rho_{24} \ddot{\theta}_{2,1}\right)$. Similarly, substituting the

\begin{tabular}{|c|c|}
\hline $\begin{array}{l}{ }^{3} \mathbf{r}_{3,1}={ }^{3} \mathbf{R}_{2}\left[r_{3} C \beta_{3,1}, r_{3} S \beta_{3,1}\left(r_{4}-r_{5}\right) / 2\right]^{T} \\
{ }^{8} \mathbf{r}_{8,1}={ }^{8} \mathbf{R}_{2} r_{8}\left[C \beta_{8,1}, S \beta_{8,1}, 0\right]^{T} \\
{ }^{9} \mathbf{r}_{9,1}={ }^{9} \mathbf{R}_{2} r_{9}\left[C \beta_{9,1} S \beta_{9,1}, 0\right]^{T} \\
\left.{ }^{2} \mathbf{r}_{2,1}={ }^{2} \mathbf{R}_{1} r_{2}[0,1,0]\right]^{T} \\
{ }^{4} \mathbf{r}_{4,1}={ }^{4} \mathbf{R}_{1} r_{4}[0,0,1] \\
{ }^{5} \mathbf{r}_{5,1}={ }^{5} \mathbf{R}_{1} r_{5}[0,0,1]{ }^{T} \\
\left.{ }^{6} \mathbf{r}_{6,1}={ }^{6} \mathbf{R}_{1} r_{6}[0,0,-1]\right]^{T} \\
{ }^{7} \mathbf{r}_{7,1}={ }^{7} \mathbf{R}_{1} r_{7}[0,0,-1]^{T}\end{array}$ & $\begin{array}{l}{ }^{3} \mathbf{r}_{3,2}={ }^{3} \mathbf{R}_{2}\left[r_{3} C \beta_{3,2}, r_{3} S \beta_{3,2},-\left(r_{4}-r_{5}\right) / 2\right]^{T} \\
{ }^{8} \mathbf{r}_{8,2}={ }^{8} \mathbf{R}_{1} r_{8}[0,1,0] \\
{ }^{9} \mathbf{r}_{9,2}={ }^{9} \mathbf{R}_{1} r_{9}[0,-1,0]^{T} \\
{ }^{2} \mathbf{r}_{2,2}={ }^{2} \mathbf{R}_{1} r_{2}[0,-1,0]^{T} \\
{ }^{4} \mathbf{r}_{4,2}={ }^{4} \mathbf{R}_{0} r_{4}\left[C \beta_{4,2}, S \beta_{4,2}, 0\right]^{T} \\
{ }^{5} \mathbf{r}_{5,2}={ }^{5} \mathbf{R}_{0} r_{5}\left[C \beta_{5,2}, S \beta_{5,2}, 0\right]^{T} \\
{ }^{6} \mathbf{r}_{6,2}={ }^{6} \mathbf{R}_{0} r_{6}\left[C \beta_{6,2}, S \beta_{6,2,} 0\right]^{T} \\
{ }^{7} \mathbf{r}_{7,2}={ }^{7} \mathbf{R}_{0} r_{7}\left[C \beta_{7,2}, S \beta_{7,2}, 0\right]^{T}\end{array}$ \\
\hline
\end{tabular}
force $\mathbf{f}_{2,1}$ in (32a) and $\mathbf{f}_{1,0}$ in (32g) into (32h) and relating the tendon forces $\mathbf{T}_{3,1}$ and $\mathbf{T}_{4,1}$ in terms of $T_{3,2}$ and $T_{4,2}$, we obtain

$$
-r_{3} T_{3,2}-r_{4} T_{4,2}+r_{1} T_{1,1}=b_{2}
$$

where

$$
\begin{aligned}
b_{2}= & -a_{1}\left(\left(a_{2}-s_{2}\right) S \theta_{2,1} m_{2}\left(\dot{\theta}_{1,0}^{2}+\dot{\theta}_{2,1}^{2}\right)^{2}\right. \\
& \left.-\left(a_{2}-s_{2}\right) C \theta_{2,1} m_{2} \ddot{\theta}_{1,0}-\left(a_{2}-s_{2}\right) C \theta_{2,1} m_{2} \ddot{\theta}_{2,1}\right) \\
& +\left({ }^{c} I_{1}+a_{1}^{2}\left(m_{2}+m_{3}\right)+m_{3} s_{1}^{2}+2 m_{3} s_{1}\right) \ddot{\theta}_{1,0} \\
& +\frac{\left(r_{1}+r_{2}\right)}{r_{3}}{ }^{c} I_{3}\left(\ddot{\theta}_{1,0}+\rho_{23} \ddot{\theta}_{2,1}\right)+\frac{\left(r_{1}-r_{2}\right)}{r_{4}}{ }^{c} I_{4}\left(\ddot{\theta}_{1,0}\right. \\
& \left.+\rho_{24} \ddot{\theta}_{2,1}\right) .
\end{aligned}
$$

Writing Eqs. (33a) and (33b) in matrix form becomes

Table II. Radius vectors used in Example 3. 


$$
\mathrm{AT}=\mathrm{B},
$$

where $\mathbf{T}=\left[T_{3,2}, T_{4,2}, T_{1,1}\right]^{T}, B=\left[b_{1}, b_{2}\right]^{T}$, and

$$
\mathbf{A}=\left[\begin{array}{ccc}
r_{2} & -r_{2} & 0 \\
-r_{3} & -r_{4} & r_{1}
\end{array}\right]
$$

From Eq. (33), we conclude that once tensions in the ground level tendons are specified, the forward dynamics can be uniquely determined. On the other hand, the inverse dynamics yields two linear equations in three unknowns. The general solution of tendon forces can be determined by the torque resolver method. ${ }^{12}$ Once tensions in the ground level tendons are determined, the tension of each segment of tendons can be obtained from Eq. (24b) from proximal to distal links while the bearing forces from distal to proximal links.

\section{Example 3}

Figure 7 shows the structure of Stanford/JPL finger. ${ }^{3}$ The direction of tendon forces and their positions can be respectively defined in Tables I and II. Note that $T_{3,1}=T_{8,1}, \quad T_{3,2}=T_{9,1}, \quad T_{4,1}=T_{8,2}, \quad T_{5,1}=T_{9,2}, \quad T_{6,1}$ $=T_{2,1}$, and $T_{7,1}=T_{2,2}$.

The displacement relation between base/ intermediate pulleys angles and joint angles is ${ }^{4}$

$$
\left[\begin{array}{c}
\theta_{1,0} \\
\theta_{8,1} \\
\theta_{9,1} \\
\theta_{4,0} \\
\theta_{5,0} \\
\theta_{6,0} \\
\theta_{7,0}
\end{array}\right]=\left[\begin{array}{ccc}
1 & 0 & 0 \\
0 & 1 & \rho_{38} \\
0 & 1 & \rho_{39} \\
1 & \rho_{84} & \rho_{34} \\
1 & \rho_{95} & \rho_{35} \\
1 & \rho_{26} & 0 \\
1 & \rho_{27} & 0
\end{array}\right]\left[\begin{array}{c}
\theta_{1,0} \\
\theta_{2,1} \\
\theta_{3,2}
\end{array}\right]
$$

Details of dynamic equations can be found in ref. 13. The dynamic equations governing the tension in the ground level tendons are given as below,

$$
\mathbf{A T}=\mathbf{B},
$$

where $\mathbf{T}=\left[T_{4,2}, T_{5,2}, T_{6,2}, T_{7,2}\right]^{T}$,

$$
\mathbf{A}=\left[\begin{array}{cccc}
r_{3} & -r_{3} & 0 & 0 \\
r_{8} & -r_{9} & r_{2} & -r_{2} \\
-r_{4} & -r_{5} & r_{6} & r_{7}
\end{array}\right]
$$

and

$$
\begin{aligned}
& \mathbf{B}=\left[m _ { 3 } ( a _ { 3 } - s _ { 3 } ) \left(a_{1}\left(S \theta_{2,1} \dot{\theta}_{1,0}^{2}+C \theta_{2,1} \ddot{\theta}_{1,0}\right)+\left(a_{2}-s_{2}\right)\right.\right. \\
& \left.\times\left(\ddot{\theta}_{1,0}+\ddot{\theta}_{2,1}\right)\right)+I_{3}\left(\ddot{\theta}_{1,0}+\ddot{\theta}_{2,1}+\ddot{\theta}_{3,2}\right) \\
& +r_{3}\left(-\frac{I_{4}\left(\ddot{\theta}_{1,0}+\rho_{84} \ddot{\theta}_{2,1}+\rho_{34} \ddot{\theta}_{3,2}\right)}{r_{4}}\right. \\
& -\frac{I_{5}\left(\ddot{\theta}_{1,0}+\rho_{95} \ddot{\theta}_{2,1}+\rho_{35} \ddot{\theta}_{3,2}\right)}{r_{5}}+\frac{I_{8}\left(\ddot{\theta}_{2,1}+\rho_{38} \ddot{\theta}_{3,2}\right)}{r_{8}} \\
& \left.+\frac{I_{9}\left(\ddot{\theta}_{2,1}+\rho_{39} \ddot{\theta}_{3,2}\right)}{r_{9}}\right) \cdot a_{1}\left(\left(a _ { 2 } \left(S \theta_{2,1} m_{2}+S\left(\theta_{2,1}\right.\right.\right.\right. \\
& \left.\left.\left.-\theta_{3,2}\right) m_{3}\right)+S \theta_{2,1} m_{2} S_{2}\right) \dot{\theta}_{1,0}^{2}+\left(a _ { 2 } \left(C \theta_{2,1} m_{2}+C\left(\theta_{2,1}\right.\right.\right. \\
& \left.\left.\left.\left.-\theta_{3,2}\right) m_{3}\right)+C \theta_{2,1} m_{2} s_{2}\right) \ddot{\theta}_{1,0}\right)+I_{2}\left(\ddot{\theta}_{1,0}+\ddot{\theta}_{2,1}\right)+\left(a_{2}\right. \\
& \left.-s_{2}\right)\left(S \theta_{3,2} a_{2} m_{3}\left(\dot{\theta}_{1,0}^{2}+\dot{\theta}_{2,1}^{2}\right)^{2}-C \theta_{3,2} a_{2} m_{3}\left(\ddot{\theta}_{1,0}\right.\right. \\
& \left.\left.+\ddot{\theta}_{2,1}\right)\right)+I_{8}\left(\ddot{\theta}_{2,1}+\rho_{38} \ddot{\theta}_{3,2}\right)+I_{9}\left(\ddot{\theta}_{2,1}+\rho_{39} \ddot{\theta}_{3,2}\right) \\
& +r_{2}\left(-\frac{I_{6}\left(\ddot{\theta}_{1,0}+\rho_{26} \ddot{\theta}_{2,1}\right)}{r_{6}}-\frac{I_{7}\left(\ddot{\theta}_{1,0}+\rho_{27} \ddot{\theta}_{2,1}\right)}{r_{7}}\right) \\
& +r_{8}\left(-\frac{I_{4}\left(\ddot{\theta}_{1,0}+\rho_{84} \ddot{\theta}_{2,1}+\rho_{34} \ddot{\theta}_{3,2}\right)}{r_{4}}\right. \\
& \left.-\frac{I_{5}\left(\ddot{\theta}_{1,0}+\rho_{95} \ddot{\theta}_{2,1}+\rho_{35} \ddot{\theta}_{3,2}\right)}{r_{5}}\right) \cdot 0.5\left(2 I_{1} \ddot{\theta}_{1,0}\right. \\
& +a_{1}\left(-\left(2\left(C \theta_{2,1}\right)^{2} h_{2} m_{2}+C \theta_{2,1} C\left(\theta_{2,1}-\theta_{3,2}\right) m_{3}\left(r_{4}\right.\right.\right. \\
& \left.\left.+r_{5}\right)-2\left(m_{2}+S \theta_{2,1} S\left(\theta_{2,1}-\theta_{3,2}\right) m_{3}\right) r_{6}\right) \dot{\theta}_{1,0}^{2} \\
& +\left(S\left(2 \theta_{2,1}\right) h_{2} m_{2}+m_{3}\left(C \theta_{2,1} S\left(\theta_{2,1}-\theta_{3,2}\right)\left(r_{4}+r_{5}\right)\right.\right. \\
& \left.\left.\left.+2 C\left(\theta_{2,1}-\theta_{3,2}\right) S \theta_{2,1} r_{6}\right)\right) \ddot{\theta}_{1,0}\right)+\left(a_{2}-s_{2}\right)\left(S \theta_{3,2} m_{3}\right. \\
& \left(-2 S \theta_{2,1} r_{6}\left(\dot{\theta}_{1,0}^{2}+\dot{\theta}_{2,1}^{2}\right)^{2}-C \theta_{2,1}\left(r_{4}+r_{5}\right)\left(\ddot{\theta}_{1,0}+\ddot{\theta}_{2,1}\right)\right) \\
& +2 m_{2}\left(-C \theta_{2,1}\left(h_{2}-r_{6}\right)\left(\dot{\theta}_{1,0}^{2}+\dot{\theta}_{2,1}^{2}\right)^{2}+S \theta_{2,1} r_{6}\left(\ddot{\theta}_{1,0}\right.\right. \\
& \left.\left.+\ddot{\theta}_{2,1}\right)\right)+C \theta_{3,2} m_{3}\left(-C \theta_{2,1}\left(r_{4}+r_{5}\right)\left(\dot{\theta}_{1,0}^{2}+\dot{\theta}_{2,1}^{2}\right)^{2}\right. \\
& \left.\left.+2 S \theta_{2,1} r_{6}\left(\ddot{\theta}_{1,0}+\ddot{\theta}_{2,1}\right)\right)\right)-2\left(I _ { 4 } \left(\ddot{\theta}_{1,0}+\rho_{84} \ddot{\theta}_{2,1}\right.\right. \\
& \left.+\rho_{34} \ddot{\theta}_{3,2}\right)+I_{5}\left(\ddot{\theta}_{1,0}+\rho_{95} \ddot{\theta}_{2,1}+\rho_{35} \ddot{\theta}_{3,2}\right)+I_{6}\left(\ddot{\theta}_{1,0}\right. \\
& \left.\left.\left.\left.+\rho_{26} \ddot{\theta}_{2,1}\right)+I_{7}\left(\ddot{\theta}_{1,0}+\rho_{27} \ddot{\theta}_{2,1}\right)\right)\right)\right] \text {. }
\end{aligned}
$$


Similar to Example 2, once tensions in the ground level tendons are specified, the forward dynamics can be uniquely determined. On the other hand, the inverse dynamics yields three linear equations in four unknowns. The example shows how the dynamic equations of a complex tendon-driven robotic mechanism can be derived.

\section{SUMMARY}

A systematic methodology for the dynamic analysis of tendon-driven robotic mechanisms is developed. The method uses the recursive algorithm to calculate the kinematics and dynamics of links. First, the kinematics of primary and intermediate links are computed recursively from the proximal link toward the end-effector link. Then, joint forces and moments are computed backwardly via the Newton-Euler equations. The force systems are established to evaluate the actual joint forces among the coaxial links.

At the end, three examples are used to illustrate the procedure of solving endless and open-ended types of tendon drives. In the endless tendon drive, we first solve the dynamics of links followed by the equations of motion. In the open-ended tendon drive, the dynamic force system will encounter the redundancy of tendon tension. The inverse dynamics can be solved from the end-effector level toward the base link while tendon forces and joint forces can be obtained from the base link toward the end-effector link. It is hoped that the methodology can be helpful for the dynamic analysis of complex tendon-driven robotic mechanisms

\section{NOMENCLATURE}

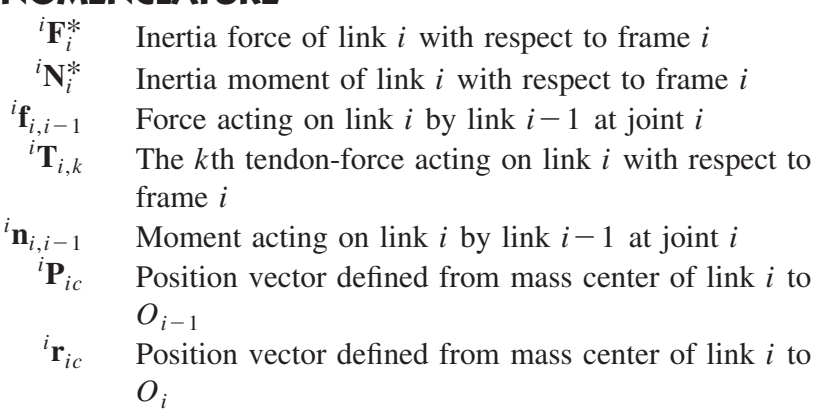

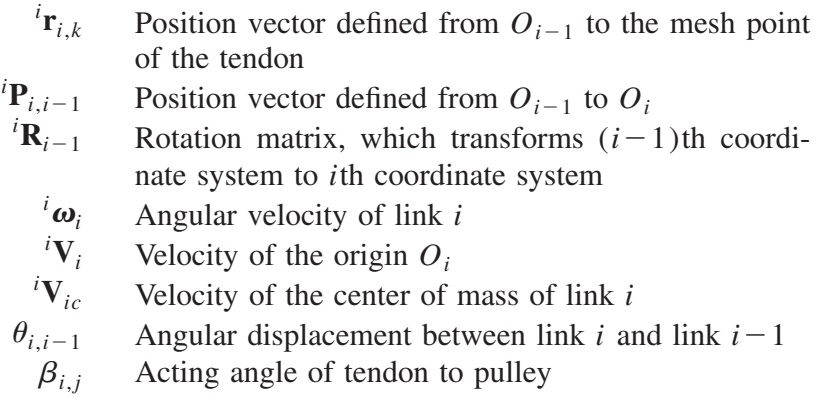

\section{REFERENCES}

1. F.Y. Chen, Mechanics of multi-pulley flexible drive systems, Mech Mach Theory 13 (1978), 643-648.

2. A. Morecki, Z. Busko, H. Gasztold, and K. Jaworek, Synthesis and control of the anthropomorphic twohanded manipulator, Proc. of the 10th Int'l Symposium on Industrial Robots, Milan, Italy, 1980, pp. 461-474.

3. J.K. Salisbury, Kinematic and force analysis of articulated hands, Ph.D dissertation, Dept. of Mechanical Engineering, Stanford University, Stanford, CA, 1982.

4. L.W. Tsai and J.J. Lee, Kinematic analysis of tendondriven robotic mechanisms using graph theory, ASME J Mech Transm Autom Des 111:(1) (1989), 59-65.

5. M.G. Hollars and R.H. Cannon, Initial experiments on the end-point control of a two-link manipulator with flexible tendons, ASME Winter Annual Meeting, Miami, FL, 1985.

6. G.M. Prisco and M. Bergamasco, Dynamic modeling of a class of tendon driven manipulators, Proc IEEE Int Conf Robotics Automat, Monterey, CA, 7-9 July 1997.

7. S.C. Jacobsen, H. Ko, E.K. Iversen, and C.C. Davis, Antagonistic control of a tendon-driven manipulator, Proc IEEE Int Conf Robotics Automat, 1989.

8. C.R. Johnstun and C.C. Smith, Modeling and design of a mechanical tendon actuation systems, ASME J Dyn Syst, Measu Control 114 (1992), 253.

9. L.W. Tsai, Robot analysis-the mechanics of serial and parallel manipulators, Wiley, New York, 1999.

10. J. Denavit and R.S. Hartenberg, A kinematic notation for lower pair mechanisms based on matrices, ASME J Appli Mech 77 (1955), 215-221.

11. D.E. Orin, R.B. McGhee, M. Vukobratovic, and G. Hartoch, Kinamatic and kinetic analysis of open-chain linkage utilizing Newton-Euler methods, Math Biosci 43 (1979), 107-130.

12. J.J. Lee and L.W. Tsai, Torque resolver design for tendon-driven manipulators, ASME J Mech Des 115:(4) (1993), 877-883.

13. Y. H. Lee, Dynamic analysis of tendon-driven robotic mechanisms, Master thesis, Department of Mechanical Engineering, National Taiwan University, Taiwan, 2002. 